\title{
Transfusionspraxis.de - Informationen für die klinische Hämotherapie
}

\author{
Transfusion Practice - a Website-based Platform for Clinical Hemotherapy
}

Autor

Institut

\section{E. K. Petershofen}

${ }^{1}$ Institut Bremen-Oldenburg, DRK Blutspendedienst NSTOB, Oldenburg

\section{Schlüsselwörter \\ - Transfusionspraxis \\ - klinische Hämotherapie \\ - Informationsplattform \\ - transfusionverantwortliche Fortbildung}

\section{Key words}

- transfusion practice

- clinical hemotherapy

- information platform

- transfusion responsible person education
Bibliografie

DOI http://dx.doi.org/

10.1055/s-0032-1328135

Transfusionsmedizin 2013; 3 :

28-30 @ Georg Thieme

Verlag KG Stuttgart · New York · ISSN 2191-8805

\section{Korrespondenzadresse} Dr. Eduard Konstantin Petershofen

Obmann der Kommission für Klinische Hämotherapie der DGTI

Institut Bremen-Oldenburg DRK Blutspendedienst NSTOB Brandenburger Str. 21

26133 Oldenburg

Petershofen@bsd-nstob.de

\section{Zusammenfassung \\ $\nabla$}

Basierend auf einer Initiative von DGTI und BDT wurde im Frühjahr 2004 die „Kommission für Klinische Hämotherapie" gebildet. Aufgabenschwerpunkt ist die Präsentation und Vermittlung von Information rund um den komplexen Bereich der „Klinischen Hämotherapie“.

Durch die Umsetzung des Transfusionsgesetzes (TFG) und die Anwendung der Richtlinien zur Hämotherapie (RiLiBÄK-Hämotherapie) besteht sowohl ein aktueller als auch sich wiederholender Informationsbedarf in diesem Bereich. Die Kommission versucht hier Abhilfe zu schaffen: einerseits durch die Internet-basierte Informationsplattform www.transfusionspraxis.de, andererseits durch das Angebot einer zentralen Fortbildungsveranstaltung im Sinne eines RefresherKurses. In diesem Jahr feiert der Kursus, der jeweils am Eröffnungstag des DGTI-Kongresses im September stattfindet, bereits sein 10-jähriges Jubiläum.

Im Jahr 2004 wurde von den Vorständen der Deutschen Gesellschaft für Transfusionsmedizin und Immunhämatologie (DGTI) und des Berufsverbands Deutscher Transfusionsmediziner (BDT) die Initiative „Kompetenz in der Klinischen Hämotherapie" gestartet.

Kernelemente dieser Initiative waren damals die Schaffung einer Internetplattform ( $\boldsymbol{O}$ Abb. $\mathbf{1})$ mit der Bereitstellung umfangreicher Informationen zum Themenbereich „Klinische Hämotherapie“. Ferner sollte ein Angebot für Transfusionsverantwortliche (TV), -beauftragte (TB), Laborleiter, aber auch für Qualitätsbeauftragte Hämotherapie und ärztliche Kolleginnen und Kollegen geschaffen werden, die sich mit Transfusionen im weitesten Sinne beschäftigen. Die Informationsseite soll kompetent informieren und denjenigen, die nicht ständig nach aktuellem Wissen im Internet su-

\section{Abstract \\ $\nabla$}

Based on an initiative of DGTI and BDT the 'Commission for Clinical hemotherapy' was founded in spring 2004.

Due to the German Transfusion Law (TFG) and the 'National Guidelines for the Use of Blood and Blood Products' (Richtlinien-Hämotherapie), a continuous need for education was registered for both education of responsible persons and continuing education for persons who are in charge for transfusion. The commission tries to support this field as much as possible with two major projects. First, an internet based website has been installed (www.transfusionspraxis.com -.eu; -.net; -.org) offering more than 1200 links for relative information, and second, organizing a central refresher course for clinical hemotherapy, which is repeated every year before the opening ceremony of the annual DGTI meeting. In 2013 the course will celebrate its 10 th anniversary.

chen können, zielführend die wesentlichen bzw. neuesten Informationen zur Verfügung stellen. In Ergänzung wird seit 2003 in Form einer ganztägigen Veranstaltung ein Fortbildungsseminar angeboten, in dem mehrere ausgewiesene Fachleute ihres Bereichs, auch aus verschiedenen medizinischen Disziplinen, fundiert und praxisnah über Alltagsprobleme ( $\boldsymbol{O}$ Abb. 2 und $\mathbf{3}$ ) und Erfahrungen berichten.

Auch in diesem Jahr wird am 24. September 2013 wieder ein Fortbildungskursus am Eröffnungstag des DGTI-Jahreskongresses, diesmal in Münster, durchgeführt. Dabei kann ein kleines Jubiläum gefeiert werden: zum 10. Mal findet diese Veranstaltung mit durchschnittlich 250-300 Teilnehmern statt.

Für die Kommission steht die Vermittlung der vorwiegend aktuellen Dinge des Alltags im Vor- 


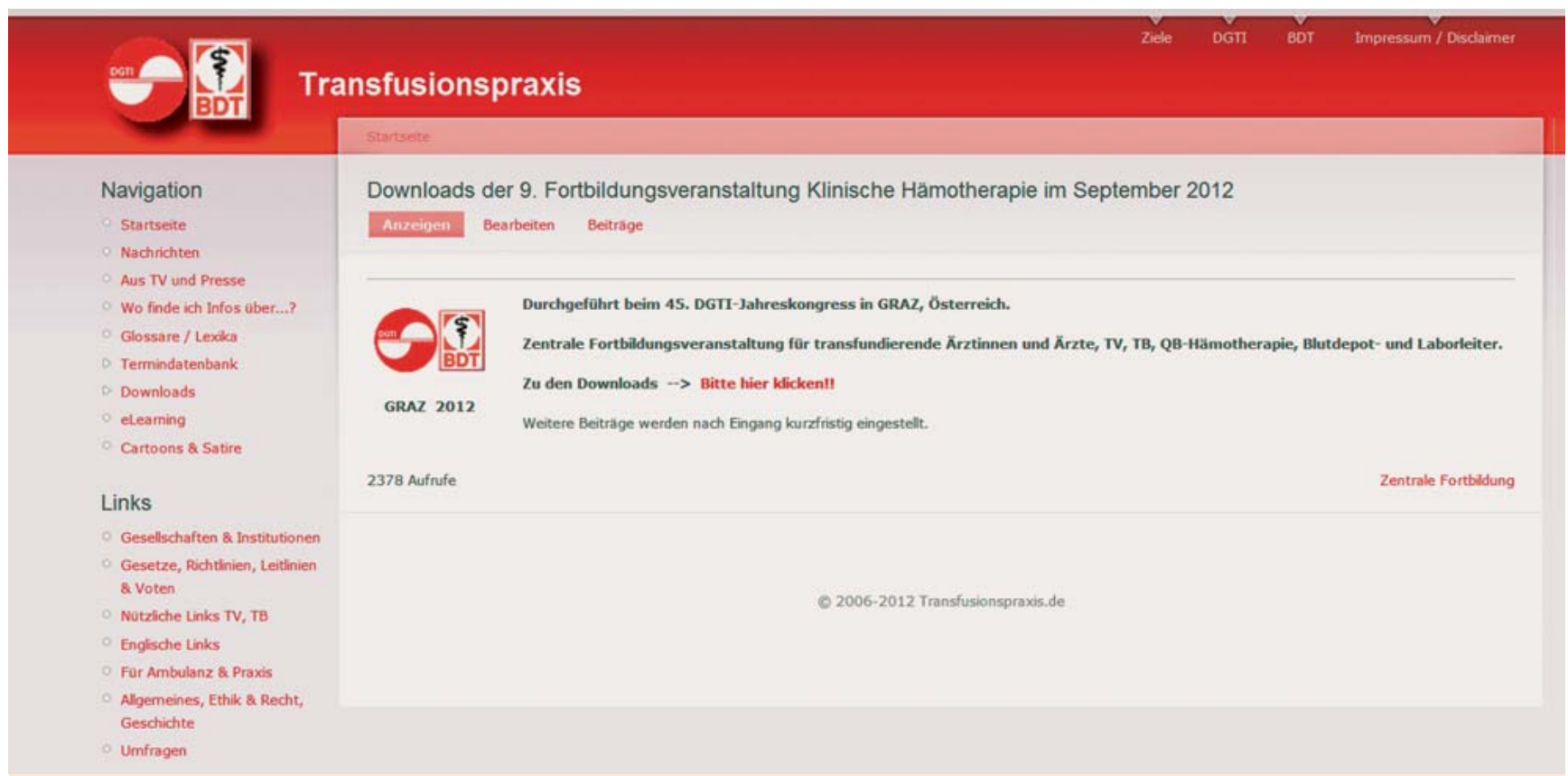

Abb. 1 Webseite der transfusionspraxis.de mit Hinweisen zum Download von Vorträgen der Fortbildung 2012.

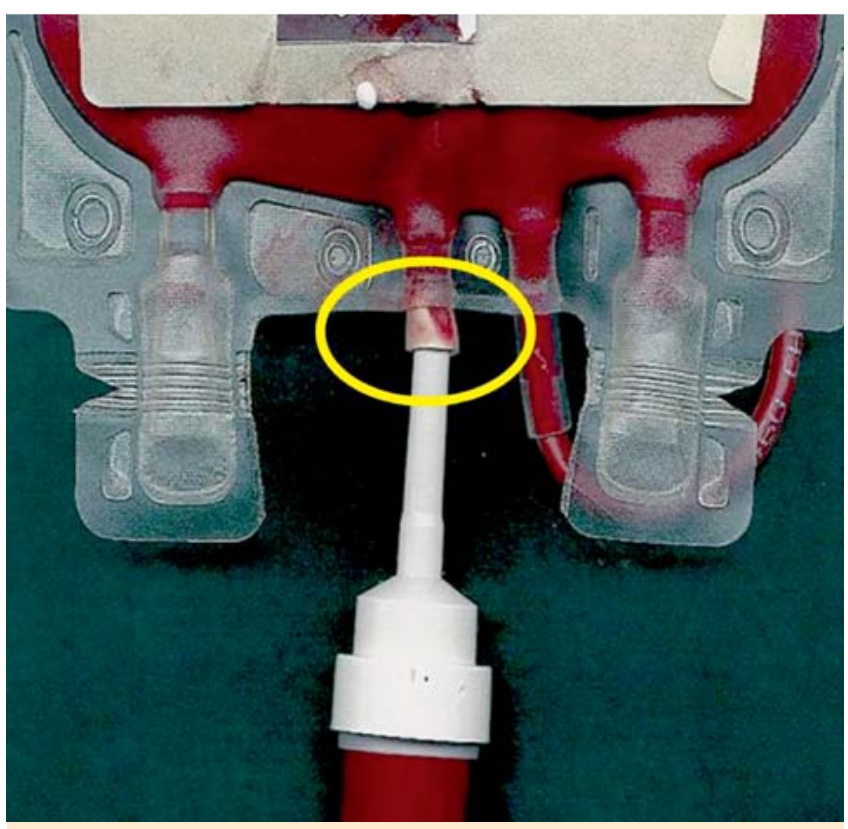

Abb. 2 Fehlerhaftes Anstechen eines Blutbeutels (nachgestellt). Risiko der Kontamination bzw. des Einströmens von Luft (Foto: E. K. Petershofen).

dergrund. Themen, wie „Welche neuen Regelungen wurden seit dem letzten Jahr umgesetzt?“, „Wie bewerkstelligen andere Institutionen die nachträgliche Aufklärung über eine stattgefundene Transfusion?“ oder „Welche rechtlichen Aspekte ergeben sich aus der Etablierung des neuen Patientengesetzes?“ sollen die $\mathrm{Zu}$ hörer animieren, eigene Fragen zu stellen.

Indikationen aus den Querschnitts-Leitlinien, QM-Vorgaben diverser Richtlinien oder mögliche Konsequenzen durch die Änderungen im Arzneimittelgesetz werden aufgezeigt und Lösungsmöglichkeiten vorgestellt. Aber auch praktische Aspekte sollen

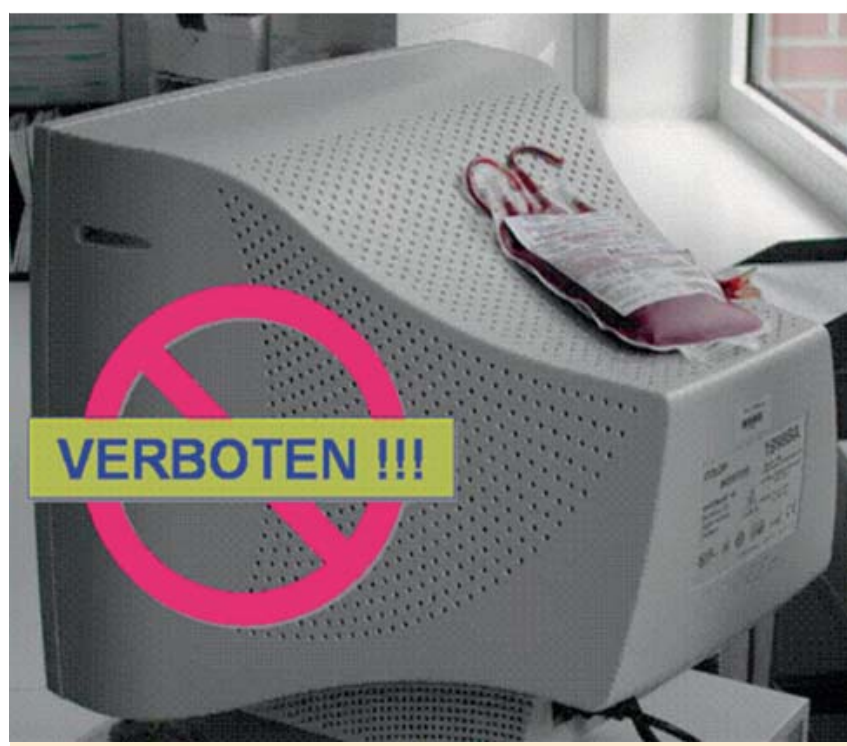

Abb. 3 Ein Bild sagt mehr als tausend Worte: Unerlaubtes Anwärmen eines Blutkonzentrats (nachgestellt) (Foto: E. K. Petershofen).

nicht zu kurz kommen (z.B. Transfusionen in der Nacht, verwechslungssichere Zuordnungen im Notfallbereich).

Als äußerst positiv hat sich dabei das Zusammenwirken verschiedener Fachgesellschaften mit Bezug zur Hämotherapie herausgestellt. In diesen Veranstaltungen werden jeweils 8 bis 9 kompetente Redner ihres Bereichs aus den Fachgesellschaften von DGTI, GTH, DGHO, DGAI ausgewählt und gebeten, ihre praktischen Erkenntnisse mitzuteilen. Die meisten Kolleginnen und Kollegen sind darüber hinaus bereit, ihre Informationen aus der Veranstaltung im Internet zur Verfügung zu stellen, damit die Zu- 
hörer auch nachträglich noch die Möglichkeit besitzen, Inhalte zu repetieren. Einen Zugang zu diesen Dateien findet man auf der Startseite der transfusionspraxis.de ( $\bullet$ Abb. 1).

Die Website stellt die 2. wichtige Säule der Initiative dar. Mittlerweile können über 1200 Verbindungen (Links) zu anderen Informationsquellen zur Verfügung gestellt werden. Die Inhalte sind jeweils nach Themengebieten geordnet und wurden sorgfältig überprüft und ausgewählt. Damit bietet die Link-Sammlung einen ausgezeichneten Überblick über das gesamte Gebiet der klinischen Hämotherapie. Des Weiteren finden sich hier Adressen, die nicht ohne weiteres über die bekannten Suchmaschinen wiedergegeben werden.

Andere wesentliche Elemente sind die Termindatenbank, die Zusammenstellung von aktuellen Gesetzen, Richtlinien, Leitlinien und Voten, die Auflistung von nützlichen Links für TV und TB sowie die Bereitstellung von Links zu PPT-Vorträgen, Referaten, Reviews und ähnlichem.
Die Termindatenbank ist so konzipiert, dass auch Außenstehende die Möglichkeit besitzen, ihre Fortbildungsveranstaltungen dort einzugeben, ggf. mit einer Datei zum Download. Die Termindatenbank bietet Ihnen einen schnellen Überblick über z.B. regional angebotene 16-Stunden-Kurse, Refresherkurse, Hinweise zu 40-stündigen QB-Kursen, aber auch zu Veranstaltungen in ihrer Nähe. Einen besonderen Service bietet der Newsletter: in unregelmäßigen Abständen werden per E-Mail aktuelle Informationen an die angemeldeten Teilnehmer verschickt. Damit sind Sie sozusagen „immer auf dem neuesten Stand“. Bei Interesse können Sie sich zu jeder Zeit anmelden, aber auch problemlos wieder abmelden.

Sollten Sie die Vorzüge der www.transfusionspraxis.de oder des Fortbildungskurses noch nicht kennen, möchten wir Sie herzlich zu einem Besuch einladen. 